\title{
Meandering jets in shallow rectangular reservoir
}

\author{
Y. Peltier, S. Erpicum, P. Archambeau, M. Pirotton \& B. Dewals \\ ArGEnCo Department, Research Group Hydraulics in Environmental and Civil Engineering (HECE), \\ University of Liège, Liège, Belgium
}

\begin{abstract}
In this article, meandering flows in a shallow rectangular reservoir are experimentally and numerically investigated. Two experiments were performed in a smooth shallow horizontal flume and the surface velocity fields were measured by Large-Scale PIV (LSPIV). The flow conditions were chosen in such a way that the friction regime of both flows was different. These flows were then modelled using the academic code WOLF2D, which solves the $2 \mathrm{D}$ shallow water equations and uses a depth-averaged $\mathrm{k}-\varepsilon$ model for modelling turbulence. The main characteristics of the measured and simulated flows were finally extracted from a Proper Orthogonal Decomposition (POD) of the surface velocity fields and depthaveraged velocity fields respectively and were compared. When the mean fluctuating kinetic energy of the considered POD mode is greater than $1 \times 10^{-5} \mathrm{~m}^{2} / \mathrm{s}^{2}$, the numerical modelling and the experiments are in good agreement whatever the friction regime of the flow.
\end{abstract}

\section{INTRODUCTION}

Shallow rectangular reservoirs are common structures in the field and they are used for water storage or sediment trapping. Their optimal exploitation strongly depends on the accurate prediction of the flow patterns that develop inside. Previous studies emphasized that, depending on the shape factor, SF $=L / \Delta B^{0.6} b^{0.4}$ ( $L$ the reservoir length, $b$ the width of the inlet channel and $\Delta B$ the width of the sudden expansion), and on the Froude number at the inlet, $\mathrm{F}=U_{\text {in }} / \operatorname{Vg} H\left(U_{\text {in }}\right.$ the velocity at the inlet, $H$ the mean water depth in the reservoir), different types of flow occur (Peltier et al., 2014a):

- Symmetric with a straight central jet $(S F \leq 6.2$ and $F<0.21)$,

- Asymmetric with the central jet impinging one or several times the lateral wall of the reservoir $(\mathrm{SF} \geq 8.1)$.

- $\quad$ Meandering $S F \leq 6.2$ and $F>0.21$.

The existence of meandering flows was recently highlighted by Camnasio et al. (2012) and Peltier et al. (2014a). This flow is characterized by spatial and temporal periodical oscillations of the central jet from the beginning to the end of the reservoir. The meandering of the jet is the hallmark of the presence of large-scale vortices in the flow, which transfer momentum from the jet towards the rest of the reservoir and induce changes in the velocity distribution, as well as in the sediment transport, compared to a configuration without meandering jet (Peltier et al., 2013). The wave-length of the meander is proportional to the width of the reservoir and/or to the friction coefficient, while the lateral spreading of the jet is affected by the shallowness of the flow, i.e. it decreases with increasing friction number, $S=f \Delta B / 8 H$ ( $f$ the Darcy-Weisbach friction coefficient) (Chu et al., 1983).

Although the physics of the symmetric and asymmetric configurations are now relatively well understood (Mullin et al., 2003, Canbazoglu and Bozkir, 2004, Kantoush et al., 2008, Dufresne 
et al., 2010, Camnasio et al., 2011) and the flow modellers have successfully demonstrated that these flow features can be reproduced using an operational numerical model, where only the friction modelling is adjustable (Dewals et al., 2008, Dufresne et al., 2011, Peng et al., 2011, Camnasio et al., 2013, Khan et al., 2013), many questions remain regarding meandering flows.

In the present article, two meandering flows are experimentally and numerically investigated. The experimental setup is first described. Information is then given on the operational numerical model WOLF2D and the k- $\varepsilon$ model we used. A Proper Orthogonal Decomposition (POD) is used for describing the transient behaviour of the jet in the description. The POD analysis enables the comparison of spatial, temporal and energetic characteristics of the experimental and numerical modelling. The results are finally discussed.

\section{MATERIAL AND METHOD}

\subsection{Experimental set-up}

The experiments were performed without sediments in a flume located in the hydraulic laboratory of the research group HECE of the University of Liège (ULg), Belgium. The experimental device consists of a horizontal glass channel of $10.40 \mathrm{~m}$ long and $0.98 \mathrm{~m}$ wide, in which movable blocks were placed to build the rectangular reservoir (Figure 1).

For these experiments, the inlet channel was $2.00 \mathrm{~m}$ long and $0.08 \mathrm{~m}$ wide. The reservoir length was set to $1.00 \mathrm{~m}$ and the width to $0.98 \mathrm{~m}$. The outlet channel was 1.50 long with the same width as the inlet channel and a tailgate ends the flume. All the surfaces were made of glass, except for the bottom of the flume (polyvinyl chloride) and the converging section (metallic sheets).

The discharge, $Q$, was measured with an electromagnetic flow-meter (uncertainty of $0.025 \mathrm{~L} / \mathrm{s}$ ). The mean water depth, $H$, in the reservoir was measured using an ultrasonic probe and the uncertainty was estimated to be equal to $1 \%$ of the mean value. The flow dynamics was video-recorded using a high-resolution camera at a rate of $25 \mathrm{~Hz}$ during 6'30' and the surface velocity fields were calculated by LSPIV (Hauet et al., 2008, Kantoush and Schleiss, 2009). The uncertainty on the mean surface velocity was estimated to $5 \%$.

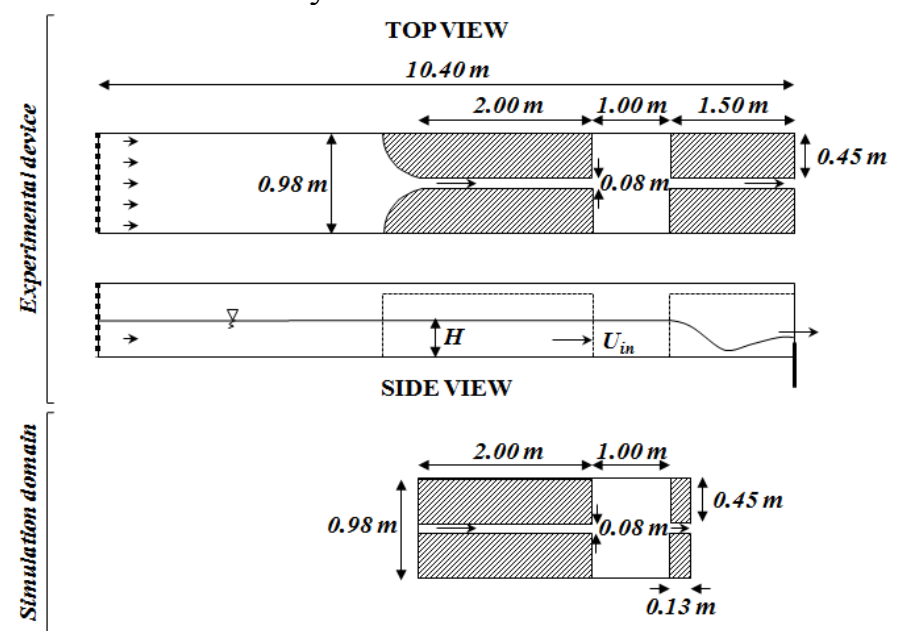

Figure 1. Sketch and pictures of the experimental device and the simulation domain.

In the present paper, $x, y$ and $z$ denote the longitudinal, the lateral and the vertical directions respectively of the Cartesian reference frame attached to the flume; $x=0$ immediately downstream from the inlet channel and $y=0$ at the right bank of the reservoir. $z=0$ at the bottom of the reservoir.

Two discharges were used, resulting in two different mean water depths in the reservoir. The corresponding Froude number, $\mathrm{F}$, friction number, $\mathrm{S}$, and Reynolds number, $\mathrm{R}=U_{\text {in }} D / v(D$ the hydraulic diameter of the inlet channel and $v$ the kinematic viscosity of the water at $20^{\circ} \mathrm{C}$ ), in the inlet channels are summarized in Table. 1. The Froude numbers of both experiments are 
close to 0.4 and are a direct consequence of the geometry of the reservoir and of the downstream boundary condition. The friction numbers are characteristic of two different friction regimes. Referring to the work of Chu et al. (2004), the flow-case F belongs to the frictional regime (the turbulence scale is mainly driven by the water depth), while the flow-case NF belong to the nonfrictional regime (the turbulence scale is mainly driven by the horizontal length-scale). The Reynolds numbers in both cases confirm that the flows are turbulent, but they are hydrodynamically smooth.

Table. 1. Main characteristics of the measured flows.

\begin{tabular}{cccccc}
\hline Test ID & $Q(\mathrm{~L} / \mathrm{s})$ & $H(\mathrm{~cm})$ & $\mathrm{F}$ & $\mathrm{S}$ & $\mathrm{R}$ \\
\hline $\mathrm{F}$ & 0.50 & 1.80 & 0.41 & 0.10 & 8,456 \\
$\mathrm{NF}$ & 1.00 & 4.20 & 0.46 & 0.03 & 24,267 \\
\hline
\end{tabular}

\subsection{Numerical model: WOLF2D}

The numerical modelling of the flows was performed using the flow model WOLF2D developed at the University of Liege (Dewals et al., 2008). It is an operational code used for engineering studies, as well as research applications.

The space discretization of the 2D-H shallow water equations was performed by means of a Finite Volume scheme. The variable reconstruction at cells interfaces was linear and was combined with a slope limiter, leading to second-order space accuracy. The convective fluxes were computed by a Flux Vector Splitting (FVS) method developed by Erpicum et al. (2010), which requires low computational cost, is completely Froude-independent and facilitates a satisfactory balance with the discretization of the bottom slope term.

The time integration was performed by means of a 3-step third-order accurate Runge-Kutta algorithm, limiting the numerical dissipation in time that smears the dynamics of the vortex generation in the jet. For stability reasons, the time step was constrained by a CourantFriedrichs-Lewy condition based on gravity waves and set to 0.2 .

The Darcy-Weisbach standard formulation was used for the friction modelling (Dufresne et al., 2011) and the bottom roughness was set to $k_{s}=0.1 \mathrm{~mm}$ (representative of the polyvinylchloride state during the experiments).

A depth-averaged k- $\varepsilon$ model with two different length-scales accounting for vertical and horizontal turbulence mixing was used (Erpicum et al., 2009). The horizontal turbulence mixing was classically modelled using the two additional transport equations $\mathrm{k}$ and $\varepsilon$, while the vertical turbulence mixing was treated with an algebraic model (Fisher Model: $v_{t}=0.08 \mathrm{Hu}^{*}, v_{\mathrm{t}}$ the turbulent eddy viscosity and $u^{*}$ the friction velocity).

In the simulations, the inlet channel of $2 \mathrm{~m}$ was reproduced in order to have an injection in the reservoir as close as possible to the experimental configuration (Figure 1). The experimental discharges were used as inflow conditions at the beginning of the inlet channel, but the unit discharge was not uniformly distributed. As prescribed by Dewals et al. (2008), the unit discharge distribution must be slightly disturbed for introducing an asymmetry in the flow, which will generate the oscillation of the jet. The disturbance linearly varied from $-1 \%$ to $1 \%$ across the inflow cells. The water depth measured $13 \mathrm{~cm}$ downstream from the outlet of the reservoir was prescribed as downstream boundary condition.

The simulation was performed in two steps. (i) a periodic regime was reached after a run of 3000 s. (ii) The last time step of the first simulation was then used as initial and boundary conditions for the second simulation, which was recorded during $500 \mathrm{~s}$ at $25 \mathrm{~Hz}$ in order to be compared with experiments.

\subsection{Proper Orthogonal Decomposition (Holmes et al., 2012)}

The meandering of the jet is characterised by spatial and temporal periodical oscillations (Figure 2 ), which are the hallmarks of the contribution of different types of coherent structures convected in the flow (Peltier et al., 2014a, Peltier et al., 2014b). Given the unsteady nature of the flows, the classical parameters (velocity fields, Reynolds shear stresses) usually used for 
describing the flows are not sufficient here for comparing experiments and numerical modelling. A method is needed for describing the unsteady characteristics of the flow.
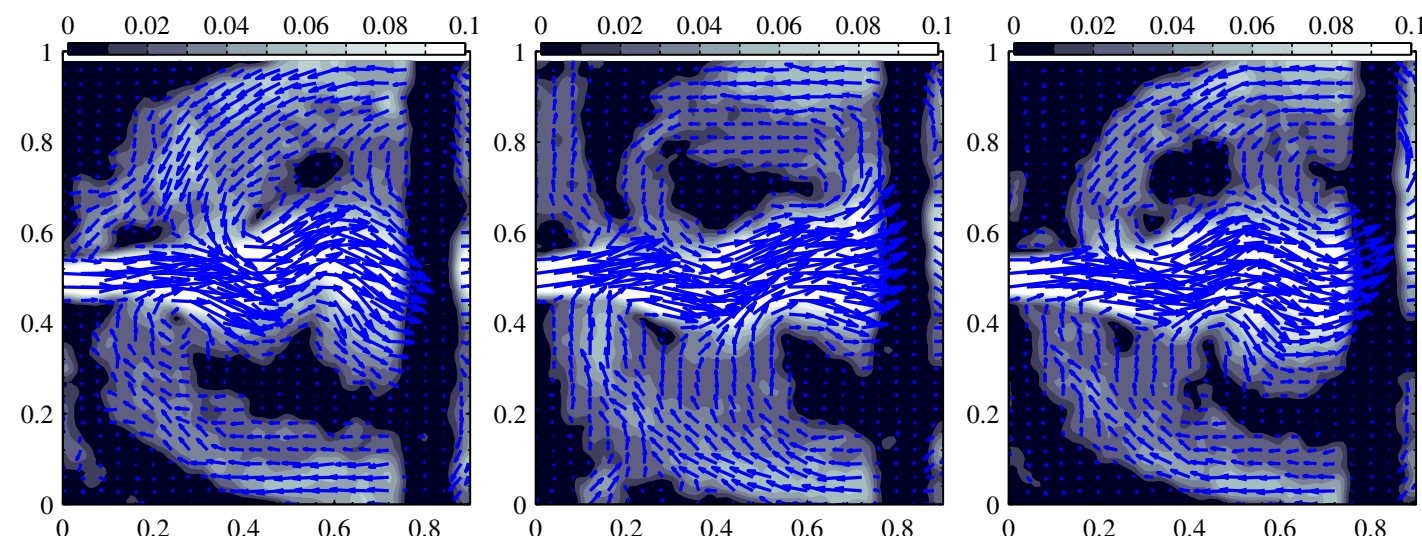

Figure 2. Experimental instantaneous velocity fields of flow-case F. The black area around $x=0.8 \mathrm{~m}$ is a blank zone in the measurements.

Considering a collection of $N$ snapshots of the fluctuating horizontal velocity fields, $\underline{u}^{\prime}\left(x_{p}, y_{p}, t_{n}\right)=\underline{u^{\prime}}(\underline{x}, t) \quad\left(n, p \in \mathbb{N}^{*}, \Delta t=t_{n+1}-t_{n}=C S T\right.$ and $\left.\underline{x} \in \Omega \subset \mathbb{R}^{2}\right)$, which are square integrable functions (i.e. $\underline{u}^{\prime}(\underline{x}, t) \in L^{2}(\Omega)$ ), the Proper Orthogonal Decomposition (POD) provides an orthonormal basis of $M$ spatial functions $\phi_{m}(\underline{x})$ of $L^{2}(\Omega)$, called spatial modes, and an orthogonal basis of $M$ temporal coefficients, $a_{m}(t)\left(m \in\{1, \ldots, \mathrm{M} \leq \mathrm{N}\}\right.$ and $\left.M, N \in \mathbb{N}^{*}\right)$, such that:

$$
\min \left(\frac{1}{N} \sum_{n=1}^{N}\left\|\underline{u^{\prime}}\left(\underline{x}, t_{n}\right)-\sum_{m=1}^{M \leq N} a_{m}\left(t_{n}\right) \underline{\phi_{m}}(\underline{x})\right\|_{L^{2}}^{2}\right)
$$

\|\|$_{L^{2}}$ being the induced norm in $L^{2}(\Omega)$ (i.e. the root mean square of the inner product for $L^{2}(\Omega)$ ).

The POD proceeds in three steps.

1) The temporal correlation matrix $C$ is first calculated:

$$
C_{i j}=\sum_{p=1}^{P} \underline{u}^{\prime}\left(\underline{x}_{p}, t_{i}\right) W_{k k} \underline{u}^{\prime}\left(\underline{x}_{p}, t_{j}\right) \quad C \in \mathbb{R}^{N \times N}, W \in \mathbb{R}^{P \times P}
$$

$W$ is a diagonal weighting matrix, for which the elements along the diagonal are the cell volumes of each of the $P$ grid points of one snapshot.

2) The temporal coefficients $a_{m}(t)$ are secondly deduced from the resolution of the following eigenvalue problem with the eig function of Matlab:

$$
\frac{1}{N} \sum_{j=1}^{N} C_{i j} \alpha_{m}\left(t_{j}\right)=\lambda_{m} \alpha_{m}\left(t_{i}\right)
$$

$C$ is definite, positive and symmetric. As a consequence, the eigenvalues $\lambda_{m}$ are all real with $\lambda_{1} \geq \lambda_{2} \geq \ldots \geq \lambda_{N}>0$, and the eigenvectors $\alpha_{m}(t)$ are orthonormal. The temporal coefficients, $a_{m}(t)$, are function of the eigenvectors and of the eigenvalues and they are orthogonal:

$$
a_{m}(t)=\sqrt{N \lambda_{m}} \alpha_{m}(t) \text { with }\left\langle a_{n}\right\rangle_{N}=0 \text { and }\left\langle a_{n} a_{m}\right\rangle_{N}=\lambda_{n} \delta_{n m}
$$

3) The spatial modes are thirdly computed by projecting the fluctuating velocity ensemble onto the temporal coefficients, i.e.:

$$
\underline{\phi_{m}}(\underline{x})=\frac{1}{N \lambda_{m}} \sum_{n=1}^{N} \underline{u}^{\prime}\left(\underline{x}, t_{n}\right) a_{m}\left(t_{n}\right) \text { with }\left\|\underline{\phi}_{m}\right\|_{L^{2}}^{2}=\underline{\phi}_{m}^{T} W \underline{\phi}_{m}=1
$$

The spatial modes are orthonormal with respect to the inner product in $\mathrm{L}^{2}, \phi_{m}{ }^{T} W \underline{\phi}_{m}$. 


\section{RESULTS AND DISCUSSION}

The POD of the experimental and numerical fluctuating velocity fields allows us to describe unsteady flows by analysing the behaviour of the first spatial modes and temporal coefficients, which are representative of the most energetic coherent structures. The POD was applied on 9,000 fields/snapshots of each measurement and numerical simulation of the flow-cases presented in Table. 1.

\subsection{Energy}

By construction of the POD basis, the $m^{\text {th }}$ eigenvalues calculated with Equation (3) correspond to the mean fluctuating kinetic energy per unit mass captured by the $m^{\text {th }}$ mode, $E_{m}$, and the sum of the $N$ non-zero eigenvalues is equal to the mean total fluctuating kinetic energy per unit mass, $E_{T}$. The mean fluctuating kinetic energy contained in the $m^{\text {th }}$ modes is plotted in Figure 3 and is compared with the simulations. For both cases, the numerical model is able to capture the energy of the first and second modes. For the frictional case F, the third and the fourth modes are paired in the simulation while it is not the case in the experiments, but the energy levels are still in good agreement. From the fifth modes the numerical model underestimated the energy. For the non-frictional case NF, the level of energy from the third mode to the sixth mode of the simulation is similar to the experiment. From the seventh the simulation fails to reproduce the energy distribution and very quickly underestimates the energy level, which indicates that the small random structures with low energy cannot be well rendered by the model.

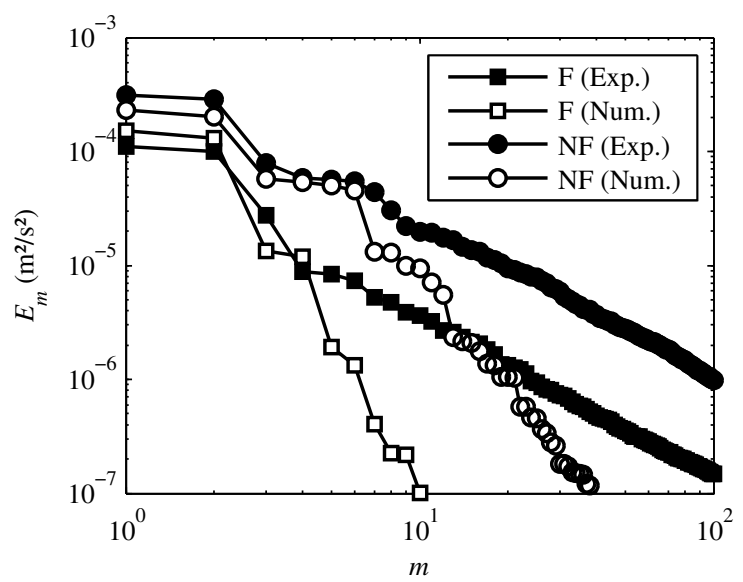

Figure 3. Mean fluctuating kinetic energy contained in the $m^{\text {th }}$ mode.

The computation of the total mean fluctuating kinetic energy indicates that for the frictional case, $E_{\text {Tnum }}$ is equal to $86 \%$ of $E_{\text {Texp }}$, while for the non-frictional case $E_{\text {Tnum }}$ is only equal to $49 \%$ of $E_{\text {Texp }}$. This indicates that the model well captured the essential structures for representing the energy in the F-case, while for the NF-case, some essential structures are missing. This is due to the energy drop between modes 2 and 3 in the F-case compared to the NF-case.

\subsection{Temporal coefficients}

The temporal coefficients were calculated with Equation (4) and they are represented for the four first modes for both flow-cases in Figure 4. For the frictional flow case, mode 1 and mode 2 of the experiment have the same patterns and are phase-shifted in time, which indicates that both modes are related to the same structures (Rempfer and Fasel, 1994). The numerical model well represents this behaviour. When considering the third and the fourth modes, no pairing is observed for experiments, while a clear pairing is obtained with the numerical model.

For the non-frictional case NF, a pairing is observed for the four first modes of the experiment and simulation. The complex patterns of the temporal coefficients are well 
represented for the two first modes. For modes 3 and 4, the simulated patterns are simpler, but numerical and experimental frequencies are close.

The numerical model cannot represent modes greater than 4 for F-case, modes greater than 6 for NF-case. Linking the temporal coefficients with the energy indicates that modes with an energy $E_{m}$ smaller than $1 \times 10^{-5} \mathrm{~m}^{2} / \mathrm{s}^{2}$ cannot be well rendered by the numerical model. These low energy modes are parasite motions due to measurement errors or high frequency motions. They have low incidence on the main flow feature (Peltier et al., 2014b).
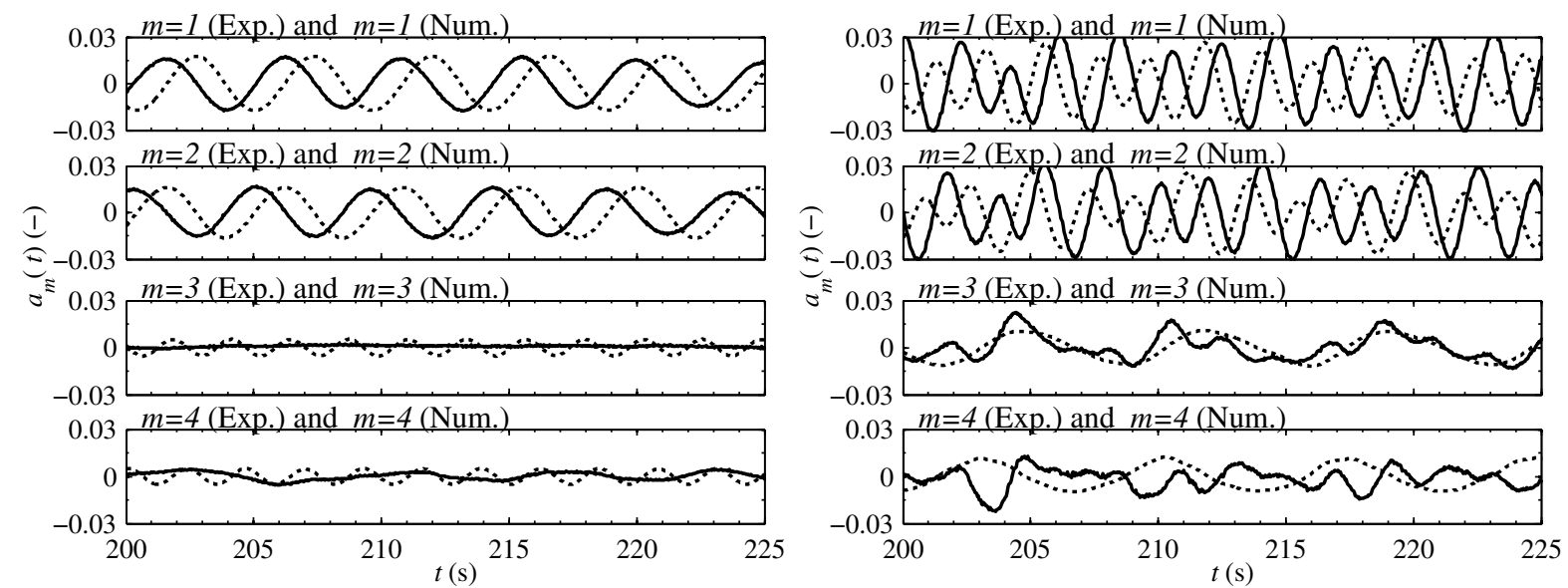

Figure 4. Temporal coefficients of the fourth first modes of the POD analysis. Plain lines correspond to experiments and dotted lines to simulations. (left figure) flow-case F. (right figure) flow-case NF.

\subsection{Spatial modes}

The vorticity of the spatial modes calculated with Equation (5) were used for describing the rotating nature of the meandering flows. Seven transversal profiles of vorticity are plotted in Figure 5 for each flow case (experiments and simulations) and the longitudinal distribution of the vorticity long the reservoir centreline is displayed in Figure 6.

For the frictional-case the numerical model is able to reproduce the lateral distribution of the vorticity of the two first modes until $40 \mathrm{~cm}$ downstream from the reservoir inlet. Downstream from this point, the numerical model fails at representing the distribution; some extreme values are indeed simulated. Nevertheless, the meander oscillations along the centreline reservoir are well represented. Concerning mode 3 and mode 4, the model completely fails at representing the spatial modes.

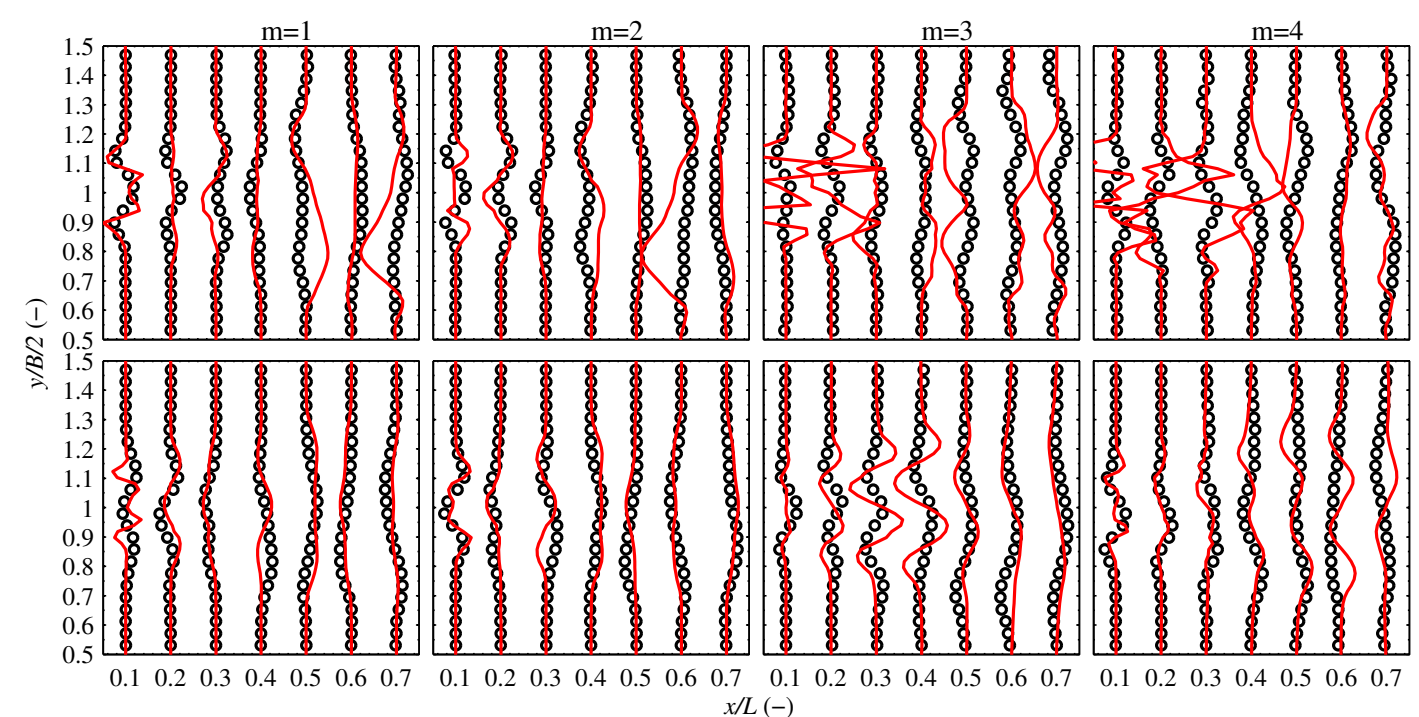

Figure 5. Lateral profiles of vorticity of the spatial modes; o experiments, - simulations. (Top figures) Fcase. (Bottom figures) NF-case. 
The numerical model captures better the physics of the non-frictional flow. Except at some locations for the third mode, the model and the experiments are in good agreement. The computed lateral distribution of vorticity as well as the longitudinal one are in a good agreement with the experiments.
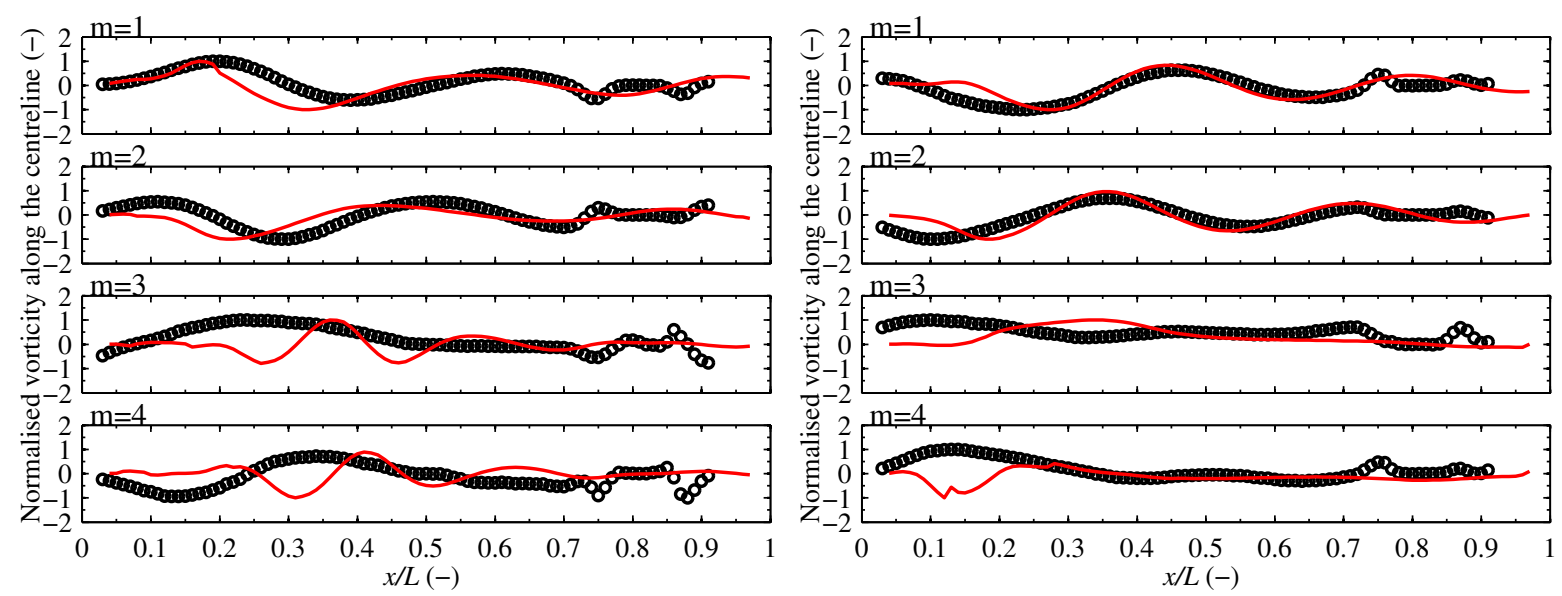

Figure 6. longitudinal profile of vorticity of the fourth first spatial modes; o experiments, - simulations. (Left figure) F-case. (Right figures) NF-case.

The previous observations are consistent with the findings obtained for the temporal coefficients. When a spatial mode corresponds to a mode with low energy, the numerical model fails to reproduce it accurately. As the non-frictional flow is more energetic than the frictional flow, and has more modes with energy greater than $1 \times 10^{-5} \mathrm{~m}^{2} / \mathrm{s}^{2}$, the overall characteristics of the non-frictional flow-case are better reproduced.

\section{CONCLUSION}

The present paper investigates experimentally and numerically two meandering flows in shallow rectangular reservoir. In the experiments, the reservoir boundary conditions were chosen to obtain flows with different friction regimes (frictional and non-frictional, see Chu et al. (2004)) and the flow dynamics was quantified by LSPIV. The flows were then modelled using WOLF2D, which solves the shallow water equations and uses a depth-averaged $\mathrm{k}-\varepsilon$ turbulence model. The numerical boundary conditions were taken as close as possible to the experiments.

A Proper Orthogonal Decomposition was performed on the measured and simulated fluctuating velocity fields for describing the main flow features. The POD indeed discriminates the structures in the flow in terms of their relative contributions to the mean total fluctuating kinetic energy and enables the description of unsteady flows.

The numerical model is able to represent POD modes, which have a mean fluctuating kinetic energy at least greater than $1 \times 10^{-5} \mathrm{~m}^{2} / \mathrm{s}^{2}$, whatever the friction regime of the modelled flow. This corresponds generally to the two to four first modes of the POD.

\section{ACKNOWLEDGMENT}

The research was funded by the University of Liège (grant SFRD-12/27). The authors are grateful for the assistance provided by the research technicians during the experiments and the fruitful discussions about the POD analysis with Prof. Vincent Denoël. 


\section{REFERENCES}

Camnasio, E., Erpicum, S., Orsi, E., Pirotton, M., Schleiss, A. J. \& Dewals, B. 2013. Coupling between flow and sediment deposition in rectangular shallow reservoirs. Journal of Hydraulic Research.

Camnasio, E., Orsi, E. \& Schleiss, A. J. 2011. Experimental study of velocity fields in rectangular shallow reservoirs. Journal of Hydraulic Research, 49, 352-358.

Camnasio, E., Pirotton, M., Erpicum, S. \& Dewals, B. 2012. Experimental and numerical investigation of a meandering jet in shallow rectangular reservoirs under different hydraulic conditions. $3^{\text {rd }}$ International Symposium on Shallow Flows. Iowa City, USA.

Canbazoglu, S. \& Bozkir, O. 2004. Analysis of pressure distribution of turbulent asymmetric flow in a flat duct symmetric sudden expansion with small aspect ratio. Fluid Dynamics Research, 35, 341-355.

Chu, V. H., Khayat, R. E. \& Wu, J. H. 1983. Stability of turbulent shear flows in shallow channel. 20th IAHR Congr., Sep. 5-9, Moscow, USSR. Delft, The Netherlands, IAHR.

Chu, V. H., Liu, F. \& Altai, W. 2004. Friction and confinement effects on a shallow recirculating flow. Journal of Environmental Engineering and Science, 3, 463-475.

Dewals, B. J., Kantoush, S. A., Erpicum, S., Pirotton, M. \& Schleiss, A. J. 2008. Experimental and numerical analysis of flow instabilities in rectangular shallow basins. Environmental Fluid Mechanics, $8,31-54$.

Dufresne, M., Dewals, B. J., Erpicum, S., Archambeau, P. \& Pirotton, M. 2010. Classification of flow patterns in rectangular shallow reservoirs. Journal of Hydraulic Research, 48, 197-204.

Dufresne, M., Dewals, B. J., Erpicum, S., Archambeau, P. \& Pirotton, M. 2011. Numerical investigation of flow patterns in rectangular shallow reservoirs. Engineering Applications of Computational Fluid Mechanics, 5, 247-258.

Erpicum, S., Dewals, B. J., Archambeau, P. \& Pirotton, M. 2010. Dam break flow computation based on an efficient flux vector splitting. Journal of Computational and Applied Mathematics, 234, 2143-2151.

Erpicum, S., Meile, T., Dewals, B. J., Pirotton, M. \& Schleiss, A. J. 2009. 2D numerical flow modeling in a macro-rough channel. International Journal for Numerical Methods in Fluids, 61, 1227-1246.

Hauet, A., Kruger, A., Krajewski, W. F., Bradley, A., Muste, M., Creutin, J. D. \& Wilson, M. 2008. Experimental system for real-time discharge estimation using an image-based method. Journal of Hydrologic Engineering, 13, 105-110.

Holmes, P., Lumley, J. L., Berkooz, G. \& Rowley, C. W. 2012. Turbulence, Coherent Structures, Dynamical Systems and Symmetry. Second Edition, Cambridge University Press.

Kantoush, S. A., De Cesare, G., Boillat, J. L. \& Schleiss, A. J. 2008. Flow field investigation in a rectangular shallow reservoir using UVP, LSPIV and numerical modelling. Flow Measurement and Instrumentation, 19, 139-144.

Kantoush, S. A. \& Schleiss, A. J. 2009. Large-scale piv surface flow measurements in shallow basins with different geometries. Journal of Visualization, 12, 361-373.

Khan, S., Melville, B. W., Shamseldin, A. Y. \& Fischer, C. 2013. Investigation of flow patterns in storm water retention ponds using CFD. Journal of Environmental Engineering (United States), 139, 61-69.

Mullin, T., Shipton, S. \& Tavener, S. J. 2003. Flow in a symmetric channel with an expanded section. Fluid Dynamics Research, 33, 433-452.

Peltier, Y., Erpicum, S., Archambeau, P., Pirotton, M. \& Dewals, B. 2013. Experimental and numerical investigation of meandering jets in shallow reservoir: potential impacts on deposit patterns. In Nguyen, K. D., Benoit, M., Guillou, S., Sheibani, N., Philipps, J. G. \& Pham Van Bang, D. (Eds.) THESIS 2013, Two-pHase modElling for Sediment dynamIcs in Geophysical Flows. Chatou, France, SHF EDF R\&D.

Peltier, Y., Erpicum, S., Archambeau, P., Pirotton, M. \& Dewals, B. 2014a. Experimental investigation of meandering jets in shallow reservoir. Environmental Fluid Mechanics.

Peltier, Y., Erpicum, S., Archambeau, P., Pirotton, M. \& Dewals, B. 2014b. Meandering jets in shallow rectangular reservoirs: POD analysis and identification of coherent structures. Accepted in Experiments in Fluids.

Peng, Y., Zhou, J. G. \& Burrows, R. 2011. Modeling free-surface flow in rectangular shallow basins by using lattice boltzmann method. Journal of Hydraulic Engineering, 137, 1680-1685.

Rempfer, D. \& Fasel, H. F. 1994. Evolution of three-dimensional coherent structures in a flat-plate boundary layer. Journal of Fluid Mechanics, 260, 351-375. 